\title{
L'œuvre de Maurice Carême. Une poésie à lire et à voir
}

\author{
Ágnes Tóth* \\ Université Catholique Pázmány Péter
}

Résumé : Maurice Carême (1899-1978) est un poète belge d'expression française. Nous proposons de revisiter l'appréciation qui en est parfois donnée de "poète des images » pour approfondir celle « avant tout visuelle » d'un poète qui écrit « avec des dons d'observation » (Moremans 1930 : 19) et donne, dans son intuition poétique, un contenu à voir. Le visuel, en symbiose avec le tangible, se manifeste dans l'œuvre de Maurice Carême par la fascination pour les gestes de la production graphique, picturale, voire même sculpturale. La passion pour les empreintes que laissent ces gestes, s'exprime par la présence d'une isotopie picturale, par la récurrence fréquente de mots tels que tracer, dessiner, peindre, esquisser, modeler et d'autres termes appartenant au même champ sémantique, ainsi que par l'utilisation du présentatif «voici ». En cette désignation de synthèse, le poète résume, dans une simulation de geste et de dénomination unique, le paysage et ses contours. Faire explicitement référence aux techniques picturales est un des procédés les plus visibles : ces expressions s'inscrivent dans un réseau de motifs visuels qui relèvent tout à la fois de la gestuelle de l'écriture et de la peinture. Cela conduit à ancrer chez le lecteur l'impression d'une poésie visuelle.

Mots-clés : Maurice Carême, visualité, isotopie picturale

Abstract: Maurice Carême (1899-1978) was a Belgian francophone poet. We present revisiting the appreciation that is sometimes assigned to a «poet of images » to deepen that « above all visual » of a poet who writes « with the gifts of observation » (Moremans 1930:19), and gives, in his poetic intuition, a content to see. The visual, in symbiosis with the tangible, manifests itself in the work of Maurice Carême through the fascination with the gestures of graphic, pictorial and even sculptural production. The passion for the imprints left by these gestures is expressed by the presence of a pictorial isotopy, by the frequent recurrence of words such as trace, draw, paint, sketch, model and other terms belonging to the same semantic field, as well as by the use of the presentative «there ». In this synthetic designation, the poet sums up, in a simulation of gestures und unique denomination, the landscape and its outlines. Explicitly referring to pictorial techniques is one of the most visible processes: 
these expressions are part of a network of visual patterns that relate both to the gestures of writing and painting. This leads to anchoring in the reader the impression of visual poetry

Keywords: Maurice Carême, visuality, pictorial isotopy

Le paradoxe de nos enquêtes sur l'imagination littéraire: trouver la réalité par la parole, dessiner avec des mots (...)

(Bachelard 196Ib : 5)

\section{Introduction}

Maurice Carême (1899-1978), poète belge d'expression française est parfois considéré comme un poète trop facile à comprendre, s'exprimant avec un lexique limpide et énonçant des idées accessibles à tous selon le langage simple de tous les jours. Le lecteur qui se saisit d'un de ses ouvrages et en aborde la lecture est effectivement surpris, lors du premier survol d'un poème ou d'un extrait de sa prose, par le décor qui y est immédiatement planté : le poète regarde et observe avec insistance les reliefs concrets, il fait parler la vie quotidienne et l'exprime dans son immédiateté, il relève la matérialité des objets ou des situations et entre en contact avec eux. C'est une poétique qui perçoit d'abord et approfondit ensuite différentes manières de se rendre le monde présent, ou de lui être présent. Appréhender son œuvre, c'est sans doute la saisir facilement en un acte premier, mais c'est aussi, dans un deuxième temps, décortiquer les vers de la poésie et scruter ce qui peut se cacher derrière ses mots pour faire émerger au jour la profondeur des pensées du poète et de sa présence au monde. Rejoindre ainsi le poète qui « ne jongle / Qu'avec des mots de tous les jours ».' (Carême 2012 : 65); ses mots qu'il nous laisse pour percevoir son intention poétique est le défi de cette étude d'approfondissement de son œuvre.

La critique littéraire relève les visages multiples de Maurice Carême : le poète lu par les enfants, le poète de la joie, Carême métaphysique, le poète de la tragédie et de la misère, le prosateur, le conteur, l'individualiste qui émerge à contre-courant, qui se sert de la prosodie traditionnelle, le poète de la simplicité, de la transparence. Les différentes facettes de la réputation de Maurice Carême posent sans doute des questions diverses et proposent des relectures de son œuvre si vaste. Dans notre relecture critique, nuançant les considérations ci-dessus, nous nous proposons de revisiter l'impression qui en est parfois laissée de poète des images et qui, selon son intuition poétique, donne un contenu à voir. 


\section{Le poète des images}

Lors de la parution en 1925 du premier recueil de Maurice Carême, 63 Illustrations pour un jeu de l'oie, Edward Ewbank (1889-1959), nomme dans son avant-propos, le poète «ymagier »,y explicitant son art :

C'est l'art de reproduire très exactement avec une simplicité sous laquelle on a bien de la peine à découvrir un peu de ruse, des objets ou des spectacles familiers. Un coloris spécial s'y ajoute - que l'on devrait appeler coloriage. Un coloris dont la franchise peut choquer lorsqu'on étudie les rapports des tonalités, teintes contre teintes, mais dont l'ensemble s'harmonise, chatoie et chante (Carême 1925 : VI).

Victor Moremans (1890-1973), journaliste et critique littéraire, en présentant Carême parmi les poètes du Prix Verhaeren en 1930, reprend le terme "ymagier ". ${ }^{2}$ Selon lui, le poète écrit une poésie « avant tout visuelle », « avec des dons d'observation »; il reste "ymagier », qualité par laquelle il occupe une place parmi les poètes. Une place qui, écrit Victor Moremans, "jusqu’à présent était vacante ». II caractérise la technique de Carême selon en termes picturaux : "la crudité de son coloris, la simplicité naïve de son coup de pinceau » (Moremans 1930 : 19). En 1931, la romaniste Émilie Noulet (1892-1978), dans un article paru dans le journal Beaux-Arts, remarque : "Maurice Carême est incontestablement un poète puisqu'il a le don de l'image [...] car un poète se reconnaît des mortels à l'usage spontané de l'image et des autres poètes à la qualité de ses images. Maurice Carême possède donc cette marque initiale » (Noulet 1931). Observateur, visuel dans son expression, artiste « des enluminures » (Flament/Champagne 1933: 145), «poète miniaturiste » (Bisque 1940 : 47), «peintre descriptif » (Delahaye 1969:58) sont les nominations répandues de Carême. Lors du colloque intitulé Maurice Carême ou la clarté profonde, organisé à Bruxelles en 1985 à l'honneur du poète, la poétesse Andrée Sodenkamp (1906-2004) met en avant « une vision de coloriste » dans ses poèmes (Sodenkamp 1992: 110) et le musicologue Jacques Chailley (1910-1999) souligne l'importance de l'image toujours présente dans la poésie de Carême « qui va dicter l'ensemble du poème et, par là, le travail du musicien » (Chailley 1992:60) ainsi que la mise en musique de ses poèmes. Le critique hongrois, László Ferenczi (1937-2015) insiste également sur l’importance de l'image chez Carême : ainsi, dans son essai Relire Maurice Carême, après avoir souligné la simplicité "savamment structurée » et la musicalité des vers du poète, il poursuit en accentuant la visibilité de cette poésie : «Et il y a les images... Vous voyez les images de Carême, alors qu'en général, dans la poésie moderne, vous ne voyez pas les images parce que ces images ont une autre fonction » (Ferenczi 1991: 1).

Dans les réflexions qui suivent, nous chercherons cette expression de Maurice Carême, le poète des images, l'observateur qui dans son intuition poétique donne un contenu à voir. Enclin à donner de la visualité à sa démarche poétique, Maurice 
Carême fut aussi très influencé par son entourage de peintres avec lesquels il noue des contacts d'amitié ; il emploie des gestes picturaux où ceux-ci deviennent l'expression du tangible et du regard. La présence de gestes picturaux dans la poésie de Carême, ainsi que la publication des œuvres réalisées en commun avec des peintres et d'autres artistes, témoignent d'un franchissement de frontières entre les arts, voire d'une complémentarité.

«Oui, Maurice Carême aimait les peintres (...) », ainsi le peintre Roger Somville (1923-2014) commençait la communication qu'il présenta lors du colloque Maurice Carême ou la clarté profonde (Somville 1992 : 163). Carême entretenait des contacts professionnels et amicaux avec plusieurs peintres de son époque. Parmi ces peintres, outre Roger Somville, il entretint en particulier des contacts suivis avec Henri-Victor Wolvens (1896-1977), Félix De Boeck (1898-1995), Marcel Delmotte (190I-1984), Michel Ciry (1919-2018). ${ }^{3}$

Maurice Carême accueillait souvent ses amis peintres dans sa maison blanche d'Anderlecht, ${ }^{4}$ ou bien il leur rendait visite dans le décor de leurs ateliers. En ces occasions, il approfondissait des dialogues amicaux et professionnels en relation avec les arts et recherches faites ensemble. Dans cette confiance amicale élaborée entre eux et par la fascination réciproque devant les talents artistiques d'un chacun, une interaction se réalisa entre les beaux-arts et les écrits poétiques. C'est par là, et sans doute aussi de là, que provient la présence de plus en plus nette des beaux-arts dans la conscience poétique de Maurice Carême et dont nous retrouvons l'expression dans son œuvre. Le peintre est représenté dans l'œuvre de l'écrivain au rang de topos, l'artiste peintre comme prototype du créateur. ${ }^{5}$

L'attention portée aux activités picturales s'accorde avec l'intérêt que Carême recherche dans son propre moyen d'expression: décrire le sensoriel, figurer les choses concrètes, «modeler », «façonner », « courber », « dessiner » l'espace désigné par l'énonciation. Dans l'extrait suivant du recueil Brabant qui « n'est pas seulement un livre à lire. Il est à VOIR. » (Sodenkamp 1992 : 104), et qui fut illustré par quatre peintres, ${ }^{6}$ non seulement Carême compare son activité à celle du peintre mais il y exprime en huit mots d'une extrême densité toute la sympathie qu'il lui témoigne : «Je notais, comme un peintre appose ses couleurs, / Les ombres qui riaient en passant sur mon cœur $»^{7}$ (Carême $\left.1976: 78\right)$.

\section{Donner à voir}

Dans l'avant-propos de l'œuvre de Carême, 63 Illustrations pour un jeu de l'oie (titre évocateur), Edward Ewbank, en présentant l'écriture de Carême, parle d'« un crayonnage préliminaire ». Ce que « les dessinateurs nomment un coup de crayon ", Carême le fait en poésie en « happant les choses vues avec la précision un peu brusque » (Carême 1925 : V). Ce « happement » poétique de Carême est à proprement parler la transfiguration des choses vues, de l'acte de voir en un donner à voir. Par la 
« vertu monstrative » (Dirkx $2015: 12)$ du texte littéraire, conforme à la tradition de « l'enargeia aristotélicienne » (idem: 17), l'écriture littéraire fait voir et met l'image sous les yeux ; cette potentialité de rendre perceptible dérive d'une aptitude particulière à faire surgir le réel au-devant de l'écriture pour le donner à contempler.

Il faut d'abord souligner l'importance des figures de style imagées chez Maurice Carême. Parmi celles-ci, la comparaison est la figure la plus fréquemment utilisée. L'emploi multiple de cette figure de style donne à sa poésie la richesse que peut contenir une image figurative in praesentia. «La comparution des choses » (Collot 2011 : 4I) au sein d'une image poétique, la permutabilité entre comparant et comparé est un des traits caractéristiques de l'écriture de Maurice Carême. Il en ressort une unité profonde non seulement des éléments du monde, mais du monde et du langage, une expérience alternative à se situer entre les mots et les choses. Dans une comparaison, la mise en évidence d'un objet par rapport à une référence lui transfère une note nouvelle qui vient l'enrichir dans la mesure même où les deux images ne sont pas immédiatement superposables. L'objet ainsi comparé s'ouvre subitement sur une extension insoupçonnée qui peut sembler l'éloigner de son originalité première mais qui souvent l'oriente vers des atouts restés inaperçus de son origine essentielle. Un « comme » s'instaure parmi des différences dans le règne du "même », dans une «représentation double " (Riffaterre 1979: 176). Cette représentation intentionnelle dans sa réalisation rapproche souvent des sensations différentes. Comme dans l'extrait du poème Rien n'a changé du recueil De plus loin que la nuit où le comparant, "une bille » tactile, colorée, transparente, ronde fait que la parole se fonde dans le visuel et le tangible: «Et le bonheur est tellement proche / Que tu peux le tenir comme une bille en poche? (Carême 1992: 109).

Le visuel, dans un ensemble avec le tangible, se manifeste dans l'œuvre de Carême par la fascination pour les gestes de la production graphique, picturale et même sculpturale. La passion pour les empreintes que laissent ces gestes, s'exprime par la présence d'une isotopie picturale, par la récurrence fréquente de mots tels que tracer, dessiner, peindre, inscrire, graver, rayer, effacer, crayonner, esquisser, modeler et d'autres termes appartenant au même champ sémantique. Faire explicitement référence aux techniques picturales est un des procédés les plus visibles : ces expressions s'inscrivent dans un réseau de motifs visuels qui relèvent tout à la fois de la gestuelle de l'écriture et de la peinture. Cela conduit à ancrer chez le lecteur l'impression d'une représentation instantanée. Décrire la scène comme un tableau, c'est ainsi la figer, suspendre son déroulement, tenter de fixer une vision.

\section{Les gestes picturaux}

Parmi les termes d'une isotopie picturale, il faut évoquer le verbe "tracer ", acte de figurer et de marquer, qui, dans l'écriture carêmienne, est souvent associé aux pas, aux orteils d'enfant, aux mains, aux pinceaux; ces démarches se retrouvent éga- 
lement dans la nature : le vent, le brochet, la buse tracent aussi des formes, ainsi que, dans un concept plus abstrait, la lumière, le bonheur.

Il semble, dès lors, intéressant de prendre en compte les différents supports de cet acte de figuration. Dans le poème Marie en Brabant du recueil Brabant, les traces sont portées par la poussière, où le profane met en lumière l'innocence divine de l'enfant.

Marie qui parle de soleil

À son enfant dont les orteils

Semblent laisser, dans la poussière,

De fines traces de lumière; (Carême 1976 : 73)

Dans l'extrait suivant du recueil Heure de grâce, la fugacité des traces de vie est exprimée dans le support du sable: «Je traverse à mon tour cette étendue de sable / Où le vent de Dieu raie toute trace de pieds » (Carême 1957 : 148). L'extrait suivant du recueil Mer du Nord fait part de l'apparition et de la disparation des traces de pas au bord de la mer, où les traces enivrées de lumière, témoignent de l'émerveillement :

Et partout des traces de pas

Remplies jusqu'au bord de lumière

S'en vont par-ci, s'en vont par-là,

Si heureuses d'être sur terre

Que le vent qui en est jaloux

Les efface parfois d'un coup. ${ }^{8}$ (Carême 1979b : 13)

Les sentiers, les ornières, « les dessins vécus » (Bachelard 1961a : 30) au fond des traces, eux aussi, gardent sur le sol et sur la végétation les empreintes laissées par le passage. Voici un exemple du recueil posthume Souvenirs, témoin, par surcroît, d’allégresse:

Marcher de l'aube au soir

En laissant, sans la voir,

La trace du bonheur

Dans la bruyère en fleur, ${ }^{9}$ (Carême 2011 : 179)

Avant d'entamer un pain, celui-ci est, par respect, signé d'une croix selon l'habitude de bénédiction des aliments; et dans l'extrait suivant du recueil Femme, le 
champ devient métonymiquement porteur du même signe et de cette même intention :

Déjà, d'un geste large, avec du beau froment,

Le premier semeur trace une croix sur son champ

Dont l'orée, près du bois, se charge d'anémones. (Carême 1946:50)

Dans le poème Le Dieu du recueil Entre deux mondes, les matériaux plus solides gardent les empreintes divines : " On trouva jusque dans les marbres / La trace de ses longs pinceaux» (Carême 1979a: 37).

Outre le sable, le sol, le champ, les marbres, l'eau est aussi un support de traces surtout éphémères. Voici en extrait du poème Bien malin! du recueil Du ciel dans l'eau:

Quelquefois un brochet passait

Scintillant comme un souvenir,

Mais si rapide à la surface

Qu'il ne laissait aucune trace

De ce que je voulais saisir. (Carême 2010 : 29)

La précarité de cette trace est renforcée par l'inversion fonctionnelle du comparant et du comparé : le comparant qui joue le rôle d' « archétype » (souvenir) est plus insaisissable que le comparé qui joue celui d' « ectype » (brochet). ${ }^{10}$ Le scintillement, même transitoire, accentue la visualité dans les vers ci-dessus. Dans le recueil L'eau passe, c'est aussi le cas dans le poème de ce même titre où la trace, étant un signe visuel, est liée au regard, lui-même à peine perceptible :

Nous avançons dans les brouillards

Devinant à peine nos frères

À cette trace de lumière

Qui reste prise en leur regard. (Carême 1952: 7)

La même image fugace est liée au miroir, qui implique un mystère préservé : un état d'entre-deux. Dans le poème Transparences du recueil Chansons pour Caprine, la volonté de désigner et de respecter l'inaccessible en tant que tel, se fait comme dans un miroir : " Je pense à ce miroir / Qui capte ton image / Sans en garder la trace. » (Carême 1930 : 40). Dans le poème À force de fixer la mer, dans le recueil Entre deux mondes, le miroir devient le lieu d'entrelacs entre l'artiste et son art : 
À force de fixer la mer,

Il finit par ne plus la voir,

Mais par se voir, lui, par se voir

Comme dans un panneau de verre. (Carême 1979a: 95)

Dans le poème suivant extrait du recueil Le sablier, l'intention de la fixation, enjeu de l'image picturale et poétique, reste ambiguë, comme le nuance le poète par l'évocation des miroirs :

Tu crois avoir fixé comme un clou le bouleau,

Arrêté sur le bois la marche d'un nuage,

Suspendu et le vent et le vol d'un oiseau.

Tu ne fus qu'alouette au jeu de vains miroirs. (Carême 1969: 47)

Il convient de compléter cet aperçu par un exemple scriptural de l'activité même du poète. Le poème Il marrive, le soir... extrait du recueil La maison blanche en fait percevoir les traces sur une page qu'il fait sienne où une force dynamique l'entraîne selon l'axe vertical de la création:

Ma page brille ainsi qu'une blancheur sans rives;

Les constellations y tracent des traits d'or.

Et les mots que j'écris, pris dans ces hautes mailles,

Semblables aux poissons des grandes profondeurs,

Éclatent brusquement avec un bruit d'écailles

Et, déchirant un monde obscurci par l'attente,

Retombent sur la terre en étoiles filantes. (Carême 1949 : 35)

À côté du verbe «tracer », les verbes directement liés à des activités picturales reviennent régulièrement dans les poèmes de divers recueils. C'est le cas du verbe " peindre »: " Je peins la verte éternité / Sur cette feuille de papier »" (Carême 1979b: 178); "Peindre mon cœur un rouge frais »'2 (Carême 2013:61) ; le cas aussi du verbe : « esquisser»: "Tu esquissais toujours le même paysage / Tout en arbres courbés au vent » (ibidem); le cas également du verbe « dessiner »: «Tu dessinais sur des écorces de platane, / Avec un éclat de charbon $»^{13}$ (Carême 2011 : 140); "De dessiner autour de moi / Un immense horizon de joie » (Carême 1974: II5) ; "Vous qui me faites dessiner, / Sur la poussière de mes livres, / Ce cœur qui me semble vibrer / Dans la clarté ? (Carême 1957 : 185) ; "Mon visage a prêté ses traits au paysage, / Mon sourire dessine à présent l'horizon » (Carême 1957 : 152). Dans l'exemple suivant, outre le verbe "dessiner", le mot «calque » renforce davantage encore le modèle pictural où deux visages s'entremêlent : celui de la mère et celui du paysage brabançon, pays d'enfance du poète. 
Sous le calque de ton visage,

Je vois déjà se dessiner

Les lignes bleues d'un paysage

Qui me fut toujours familier. ${ }^{14}$ (Carême $1976: 84$ )

Ce paysage brabançon familier émerge dans la poésie de Carême comme un repère figural : dans le recueil La maison blanche, le poème liminaire de la partie intitulé Brabant se termine avec les vers suivants : "Je voudrais conférer ta grâce et ta puissance / À ces vers modelés sur tes justes contours $»^{15}$ (Carême 1949 : 123). Le verbe «modeler » reparaît dans le poème Paysage, où le poète en fait image : « Je vous modèle à mon image, / Courbe ardente du paysage » (idem : 126).

Le substantif «crayon » et le verbe «crayonner » apparaissent également dans les poèmes. Nous citons deux exemples où l'espace se fait voir par ce procédé : l'un se réfère à la mer, tiré du recueil Mer du Nord : «L'horizon, d'un crayon précis, / A délimité son sourcil $»^{16}$ (Carême 1979b : 19). L'autre au Brabant, tiré du recueil La maison blanche:

Je te regarde, neige, effacer peu à peu

Le Brabant sobrement crayonné par l'hiver.

Je cherche à deviner la trace des ornières

Qui s'en allaient sans hâte au fond des chemins creux. ${ }^{17}$ (Carême 1949 : 136)

Le verbe «effacer » y apparaît : une procédure technique caractéristique de la maîtrise graphique. L'effacement, comme «technique » surgit également de cet extrait du recueil Volière pour rendre la figure brumeuse, une fois de plus liée aux mystères des miroirs, aux reflets fugaces:

Je suis la fille des miroirs.

Personne ne sait où je vis.

Suis-je d'ailleurs, suis-je d'ici?

Le plus indécis des brouillards

M'efface et me rend à la nuit. (Carême 1953: 48)

En dernier lieu citons, le poème IV du recueil Reflets d'hélices, une des premières œuvres de Carême parue en 1932, écrite en vers libres, de longueur différente, selon de brèves notations :

Vierges aux lignes pures,

Les rails joignent leurs longues mains

À l'horizon 
Car l'avenir s'annonce,

Clair et dur

Comme un bel hiver.

Déjà des reflets se précisent

Dans les jeux

Des miroirs.

\section{Il faudra que ma voix}

Se grave dans les mémoires. (Carême 1932 : 15)

«Lignes », « rails », « longues mains », « horizon », « reflets », « jeux des miroirs », autant d'images qui interfèrent dans une suite futuriste : formes, dynamisme, visualité et sonorité. L'attention donnée par le verbe " graver » du dernier vers, lié à l'acte créateur tangible, fait ressortir l'importance du geste dans l'écriture.

Chacune de ces expressions d'une activité par le verbe correspondant est à percevoir comme intention de dire et traduire un acte tangible et perceptible de création par une expression qui devient la voix du poète. Tracer, fixer, peindre, esquisser, dessiner, modeler, crayonner, graver deviennent l'expression de gestes animés qui donnent à l'écriture sa dimension visible et tangible.

Outre ces expressions gestuelles, la poésie de Carême est caractérisée par son objectivité. Hormis la mise en évidence de la première personne en style lyrique, l'écriture carêmienne se fait souvent discrète et dissimule l'impact personnel pour ne retenir que l'objectivité des détails. L'énumération des objets familiers au fil des poèmes sont bien là comme témoins d'une présence. Par cette figure d'amplification, le poète crée une composition du monde qui traduit la rencontre du sujet et des objets de l'environnement. Le présentatif « voici » souvent utilisé dans les poèmes comme une sorte de geste déictique - témoigne du défi de la poésie de montrer une image qui n'est pas en face de nous mais qui, au cœur d'elle-même, se donne à voir en nous y intégrant.

\section{Le geste déictique : voici}

La voix du poète laisse percevoir une image selon une visibilité qui déborde en même temps le champ du visible. L'homme est capable de désigner un objet écarté ; comme Michel Collot l'écrit, « ce geste destine le Montrant à devenir un être de langage, apte à évoquer la chose dans son absence même » (Collot 1989 : 187). Par ce geste direct de l'énonciation, la poésie désigne explicitement des éléments et pose leur existence ; et dans cette désignation, les présentatifs « c'est », « il y a », « voici », " voilà » jouent un rôle significatif. Ces structures contribuent à l'acte de la représentation : leur valeur sémantique fondamentale est de présenter et d'affirmer l'exis- 
tence ou la non-existence d'un objet ou d'un phénomène ; suivant la définition d'Alain Rabatel - leur rôle est intensifié par " une valeur énonciative de représentation, renseignant autant sur l'objet du discours présenté que sur le locuteur ou l'énonciateur à l'origine de la présentation » (Rabatel 200I: 134). Parmi ces structures, le présentatif «voici », très souvent utilisé par Maurice Carême, suscite notre intérêt. Cette formule marquée par les effets d'une opération d'intentionnalité, contribue à la « présentation / monstration » d'une relation réciproque du sujet posant et de l'objet posé (apud idem: 132).

Souvent placé au début d'un vers, "voici » introduit, dans les poèmes, une localisation temporelle ou spatiale, situe le cadre de la perception et affirme la réalité des objets et des circonstances de l'expérience perceptive. Par exemple: « Voici venir la nuit. / Le ciel a renversé / Son large sablier / Et tient tout l'infini / Captif en ses yeux gris. » (Carême $1974: 26$ ) ; "Voici déjà luire les mûres » ${ }^{19}$ (Carême $1960: 38$ ) ; "Et voici le village où tu aimerais vivre $»^{20}$ (Carême 1976:208); "Voici du pain, du lait, des fruits, des rameaux verts $»^{21}$ (idem : 209). La désignation des objets, des phénomènes, introduite par "voici ", sans verbe conjugué, met en scène la représentation d'un contact direct avec l'environnement ; l'énumération, les groupes nominaux accentuent la force descriptive de ces vers. La description n'a sa force que dans l'immédiateté et la proximité ; elle obtient ainsi une affirmation d'existence, une certification de présence.

Les deux dernières citations ci-dessus sont prises du recueil Brabant, dans lequel la description du paysage est au cœur de l'œuvre. Par «voici », le poète désigne, dans une simulation de geste unique de dénomination, le paysage et ses éléments. C'est le cas du poème Voici venir le crépuscule dans le même recueil. Le présentatif est à l'incipit du poème: le titre et le premier vers du poème introduisent, selon une valeur cataphorique, l'image du paysage, tout en nous positionnant dans ce paysage.

\author{
Voici venir le crépuscule \\ Doré de hauts \\ Dizeaux qui brûlent \\ Sur l'horizon en vol d'oiseau \\ Avec ses fumées qui ondulent \\ Sur le hameau. \\ Dans le lointain, près du ruisseau \\ Qui se dérobe, \\ Bêle un agneau. \\ Et l'on entend, sur les coteaux, \\ Glisser tout doucement la robe \\ Usée de l'aube.
}




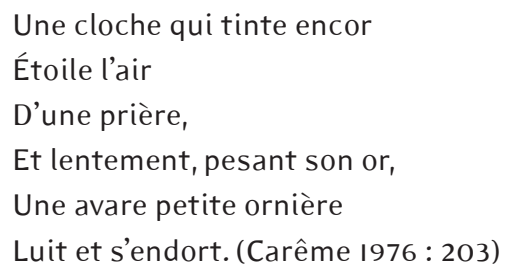

Suivi de « voici », succède une énumération d'éléments de la nature. Par la proximité immédiate de «-ci », et de «lointain » également, le poète fait voir la représentation qu'il se fait du paysage ou des objets qu'il veut mettre en évidence selon l'expérience directe qu'il en a, une perception très tangible («Glisser tout doucement la robe / Usée de l'aube », «pesant son or ») et auditive («l'on entend », « Une cloche qui tinte ») que visuelle (« doré », « sur l'horizon en vol d'oiseau », « étoile », « luit »).

Dans un autre poème dont le titre est précisément $\varepsilon$ t voici..., cette fois dans le recueil $D$ u ciel dans l'eau, ce procédé prend davantage d'importance avec la répétition du présentatif « voici»:

Et voici le soir qui revient

Avec sa douceur sur les toits,

Ses cloches tintant çà et là

Et sa brume sur les jardins.

Et voici les oiseaux qui rentrent

Avec des cris et des vols bas,

Et les fontaines dont la voix

S'élève, de plus en plus tendre.

Voici les lampes qui s’allument

Une à une dans les maisons

Dont les cheminées hautes fument

Et bleuissent sur l'horizon.

Les outils se sont endormis

Près des chariots dans l'ombre amie.

Comme les cœurs, sur leurs journées,

Les volets se sont refermés.

Même les bêtes se reposent.

C'est bien sûr dans l'ordre des choses. (Carême 20I0 : 85) 
La répétition de "voici", à plusieurs reprises dans l'énumération ci-dessus, accentue la force de la parole qui oriente et spatialise directement le champ du regard selon une vision plus vaste et jusqu'à s'en rapprocher. Dans cette désignation, non seulement le paysage et les objets cités sont thématisés, mais également le geste désignatif lui-même et le regard posé sur ce paysage pour toucher "le point de vue" (apud Rabatel 200I) élargi et rapproché et rencontrer le lecteur ainsi invité à tout voir. L'origine morphologique de l'impératif du présentatif "vois ici” insère le lecteur dans l'observation. Citant Pierre Ouellet, "voici” "s'énonce comme une invite à voir, comme l'injonction et l'ostension propres à toute prise de parole" (Ouellet 2005: 80). Le dernier vers du poème est introduit par un autre présentatif “c'est", une manière de renforcer ainsi la valeur existentielle du tableau décrit.

La reprise anaphorique de "voici" est également à la base du poème ci-dessous extrait du recueil Heure de grâce. Mais, cette fois, la désignation descriptive d'un élément d'extériorité introduit l'attention vers l'intériorité à laquelle peut conduire l'orientation amorcée.

Et la voici soudain de neige,

Cette âme toute bleue de nuit;

Voici que s'ouvre en son ennui

Le plus serein des sortilèges.

La voici de plume légère,

Cette âme lourde d'avanies;

La voici douce d'ancolie

Sous une lune de verrière.

La voici retrouvant enfin

Les lointains dorés de lumière

Où, balancée par la prière,

Elle ondulait avec le lin.

Voici qu'aux épines-vinettes

Chante le ciel des avenues

Et que, sur l'âme reconnue,

Il pleut sans fin des alouettes. (Carême 1957 : 139)

L'insistance désignative sur le paysage présente tout autant des éléments visuels que tactiles, auditifs ou émotionnels (neige, plume, ancolie, verrière, lumière, lin, épines-vinettes, chante, avenues, alouette), pour les mettre en communication avec l'âme « toute bleue de nuit », «lourde d'avanies » et " reconnue ». C'est dans 
ce cadre sensoriel que "s'ouvrent » les sortilèges et la poésie elle-même ainsi que la présentation d'une perception active et agissante par excellence. Le poème suivant, extrait du recueil Images perdues, illustre la manière de capter ces instants de perception où le vers, introduit par le présentatif « voici » placé au milieu du poème, apparaît comme une exclamation significative de sa valeur démonstrative, mettant en évidence l’image inhérente du moment.

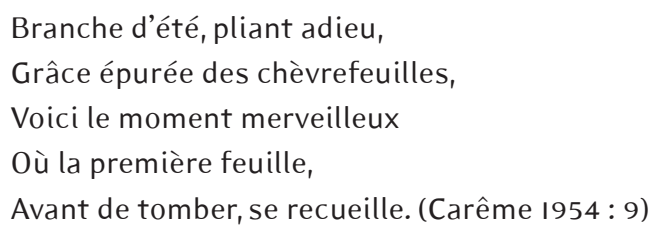

Ce geste de désignation percutante capte ainsi un moment existentiel pour le rendre perceptible tant dans sa matérialité que dans sa profondeur intrinsèque. La recherche d'une représentation possible du monde rapproche l'intention du poète et le travail du peintre qui, selon les mots de Merleau-Ponty, «n'est spectacle de quelque chose qu'en étant 'spectacle de rien', en crevant la 'peau des choses' pour montrer comment les choses se font choses et le monde monde » (Merleau-Ponty 1964 : 69).

\section{Conclusion}

Tout comme les expressions se référant aux techniques picturales, - telles que tracer, peindre, esquisser, dessiner, crayonner, graver -, témoignent d'une conviction très forte que la poésie elle-même se donne à voir, ainsi le présentatif « voici » s’inscrit dans l'énoncé poétique proche des techniques de la peinture, comme une intuition poétique pour se donner à voir. Le peintre et le poète, sont face à un même défi : faire voir, représenter et exprimer avec les moyens qui sont propres à l'art d'un chacun.

Chez Carême, nous avons relevé une prédilection de se faire accompagner par les techniques picturales pour faire vivre la matérialité, la tactilité de la création poétique. Rapprocher les techniques du poète de celles du peintre s'impose comme une démarche qui ne peut échapper à une approche de la perception, de la compréhension et de l'interprétation de l'œuvre de Maurice Carême. 


\section{Notes}

* Docteur en Littérature de l’Université Catholique Pázmány Péter de Budapest, Ágnes Tóth y soutint en 2020 sa thèse de doctorat Les représentations de l'espace et du corps dans l'œuvre de Maurice Carême. Aujourd’hui assistante au Département de Français à l’Université Catholique Pázmány Péter, elle est chargée de cours en Littérature. Ses axes de recherche portent principalement sur la littérature francophone et la poésie. En 2013, elle remporta le Prix d'études littéraires Maurice Carême, à Wavre (Belgique), ville natale du poète.

' « Jongleur, on peut l'être... » (Carême $2012: 65$ ).

${ }^{2}$ Dans les années qui suivent la parution de l'avant-propos d'Edward Ewbank, plusieurs critiques, journalistes reprendront le terme «ymagier ». À titre d’exemple : J. Conrardy dans le Journal de Bruxelles (I0/0I/1926) ; Maurice Maricel dans La Wallonie de Liège (16/0I/1926); Victor Moeremans dans La Gazette de Liège (28/OI/1926); Marc Augis dans le journal Indépendance belge (13/6/1929).

${ }^{3}$ Dans la Bibliographie parmi les références des œuvres de Maurice Carême, les peintres et dessinateurs sont mentionnés.

${ }^{4}$ Voici deux extraits des lettres amicales qui en font témoignage. La première citation est un extrait tiré de la lettre de Michel Ciry, écrite le 27 décembre 1946 à Chatou : «Laissez-moi vous remercier, bien tardivement pour la si charmante journée passée dans votre chère Maison ». L'extrait suivant est tiré de la lettre de Félix De Boeck fait le 12 septembre 1965 à Drogenbos. « Je pense régulièrement à vous et souvent l'envie me prend de pousser une pointe jusqu'à Anderlecht, mais les circonstances exceptionnelles que vous connaissez m'en empêchent. Il n'est cependant pas nécessaire de se voir fréquemment pour s'aimer et se comprendre. » Manuscrits conservés auprès de la Fondation Maurice Carême.

${ }^{5}$ « L'artiste » (Carême 1979a: 9).

${ }^{6}$ La $2^{\mathrm{e}}$ édition (Bruxelles, Arcade, 1970) fut illustrée de 7 dessins de War Van Overstraeten et 8 dessins de Hubert Malfait ; la $3^{\mathrm{e}}$ édition (Paris, Les Éditions Ouvrières, 1976) de 14 dessins de Félix De Boeck, 6 dessins de Hubert Malfait et 7 dessins de Henri-Victor Wolvens.

${ }^{7}$ « Assis à ma fenêtre » (Carême $\left.1976: 78\right)$.

8 «Vagues » (Carême 1979b: 13).

9 «Ma vie » (Carême 2011: 179).

${ }^{10}$ Parmi les anomalies structurales et fonctionnelles, c'est le cas de la redondance externe, où l'inversion de rapport archétype-ectype constitue l'anomalie, le comparant (l'archétype) et moins connu que le comparé (ectype) (apud Cohen 1968).

" «Sur ma page » (Carême 1979b : 178).

12 «La paille crue du bonheur » (Carême 2013:61).

13 « Tu dessinais... » (Carême 2011 : 140).

${ }^{14}$ « Est-ce toi, mère... » (Carême $1976: 84$ ).

15 «Brabant! dont le seul nom » (Carême 1949: 123).

${ }^{16}$ «C'est dimanche » (Carême 1979b : 19).

17 «Hiver» (Carême 1949: 136). 
18 «La fille des miroirs » (Carême $1953: 48)$.

19 «Fin d'été » (Carême 1960 : 38).

20 « Le village où tu aimerais vivre » (Carême $1976: 208)$.

${ }^{21}$ «À la santé du gel » (idem : 209).

\section{Bibliographie}

Bachelard, Gaston (1961a), La poétique de l'espace, Paris, PUF, 3e édition, [1957].

-- (196Ib), La flamme d'une chandelle, Paris, PUF.

Bisque, Anatole (1940), Préface à l'Anthologie de Poèmes Inédits de Belgique (1940), Bruxelles, Pylone.

Carême, Maurice (1925), 63 Illustrations pour un jeu de l'oie, Avant-propos d'Edward

Ewbank, Bruxelles, Édition «Revue sincère ».

-- (1930), Chansons pour Caprine, Bruxelles, Henriquez.

-- (1932), Reflets d'hélices, Bruxelles, La renaissance du livre.

-- (1946), Femme, Bruxelles, Les Éditions du Croquis.

-- (1949), La maison blanche, Paris, Éditions Bourrelier et Colin.

-- (1952), L'eau passe, Bruxelles, chez l'auteur, couverture dessinée par Michel Ciry.

-- (1953), Volière, Bruxelles, Éditions Bourrelier et Colin, couverture dessinée par

Roger Gobron.

-- (1954), Images perdues, Bruxelles, chez l'auteur.

-- (1957), Heure de grâce, Bruxelles, chez l'auteur.

-- (1960), La flûte au verger, Bruxelles, Les Éditions Ouvrières.

-- (1969), Le sablier, Paris, Les Éditions Ouvrières.

-- (1974), De feu et de cendre, Paris, Fernand Nathan.

-- (1976), Brabant, Paris, Les Éditions Ouvrières, 3e édition, illustré de 14 dessins de

Félix De Boeck, 6 dessins de Hubert Malfait et 7 dessins de Henri-Victor Wolvens, [1967].

-- (1979a), Entre deux mondes, Paris, Fernand Nathan, 4e édition, couverture dessinée par Serge Creuz, [1970].

-- (1979b), Mer du Nord, Paris, Fernand Nathan, 4e édition, dessins de Henri-Victor Wolvens, [1971].

-- (1992), De plus loin que la nuit, Bruxelles, Éditions Vie Ouvrière, Collection « Pour le plaisir », dessins de Michel Ciry. 
-- (20I0), Du ciel dans l'eau, Lausanne, Éditions de L’Âge d'Homme, Collection « La Petite Belgique».

-- (2011), Souvenirs, Lausanne, Éditions de L’Âge d'Homme.

-- (2012), Le Jongleur, Lausanne, Éditions de L'Âge d'Homme.

-- (2013), L'Évangile selon Saint Carême, Lausanne, Éditions de L’Âge d'Homme.

Chailley, Jacques (1992), " Maurice Carême vu par un musicien », In Maurice Carême ou la clarté profonde (Colloque 22-24 novembre 1985), Bruxelles, Commission communautaire française de la Région de Bruxelles-Capitale : 57-63.

Cohen, Jean (1968), «La comparaison poétique : essai de systématique », Langages, $3^{e}$ année, $n^{\circ} 12$, Linguistique et littérature, pp. 43-51, URL : https://www.persee.fr/ doc/lgge_0458-726x_1968_num_3_12_235I [consulté le 19/05/2020]

Collot, Michel (1989), La poésie moderne et la structure d'horizon, Paris, PUF.

-- (20II), La Pensée-paysage - Philosophie, arts, littérature, Paris, Actes Sud /ENSP.

Delahaye, Gilbert (1969), Maurice Carême, Tournai, Unimuse, Collection « Le miroir des Poètes ».

Dirkx, Paul (2015), « Introduction - Un œil qui passe inaperçu », in Dirkx, Paul (éd.), L'œeil littéraire - La vision comme opérateur scriptural, Rennes, Presses universitaires de Rennes, Collection «Interférences » : 9-22.

Flament, A./ Champagne, Paul (1933), Écrivains belges d'aujourd'hui, Bruxelles, Office de Publicité.

László, Ferenczi (1991), Relire Maurice Carême, essai conservé auprès de la Fondation Maurice Carême ; Prix d'études littéraires Maurice Carême.

Merleau-Ponty, Maurice (1964), L'oeil et l'esprit, Paris, Éditions Gallimard.

Moremans, Victor (1930), Les Poètes du Prix Verhaeren (1923-1930), Liège, Aux Éditions de Vigie 30.

Noulet, Émilie (1931), «Poètes belges », Beaux-Arts, Revue d'art, Bruxelles, article paru le 24/04.

Ouellet, Pierre (2005), "Quelque part - Topique et poétique », in Russo, Adélaïde, Harel, Simon (éd.), Lieux propices. L'énonciation des lieux, le lieu de l'énonciation dans les contextes interculturels, Centre d'études françaises et francophones, Québec, Presses de l'Université Laval, Collection «Intercultures »: 77-90.

Rabatel, Alain (200I), "Valeurs énonciative et représentative des 'présentatifs' c'est, il y a, voici / voilà : effet point de vue et argumentativité indirecte du récit ", Revue de Sémantique et Pragmatique, $\mathrm{n}^{\circ} 9$, Presses de l'Université d'Orléans : III-144, URL: https://halshs.archives-ouvertes.fr/halshs-0043304I [consulté le 19/05/2020]

Riffaterre, Michael (1979), La production du texte, Paris, Éditions du Seuil, Collection «Poétique».

Sodenkamp, Andrée (1992), "Maurice Carême et le Brabant ", In Maurice Carême ou la clarté profonde (Colloque 22-24 novembre 1985), Bruxelles, Commission 
Ágnes Tóth

communautaire française de la Région de Bruxelles-Capitale : 103-113.

Somville, Roger (1992), "Quelques mots au sujet de Maurice Carême », In Maurice Carême ou la clarté profonde (Colloque 22-24 novembre 1985), Bruxelles, Commission communautaire française de la Région de Bruxelles-Capitale : 163168. 